\title{
Preclinical assessment of potential interactions between botulinum toxin and neuromodulation for bladder micturition reflex
}

\author{
Xin Su ${ }^{1^{*}}$, Angela Nickles ${ }^{2}$ and Dwight E. Nelson ${ }^{1}$
}

\begin{abstract}
Background: While botulinum toxin A (BoNT-A) has become a more commonly used second-line treatment for patients with detrusor overactivity, it remains unknown whether the impacts of this therapy may persist to influence other therapies such as sacral neuromodulation. In this preclinical study we have evaluated urodynamic functions to intradetrusor injection of BoNT-A and the bladder inhibitory effects of spinal nerve stimulation (SNS) following BoNT-A treatment.
\end{abstract}

Methods: Female rats were anesthetized with $3 \%$ isoflurane. BoNT-A (2 units, $0.2 \mathrm{ml}$ ) or saline were injected into the detrusor. Rats then were housed for 2 days to 1 month before neuromodulation study. Monopolar electrodes were placed under each of the L6 spinal nerve bilaterally under urethane anesthesia. A bladder cannula was inserted via the urethra for saline infusion and intravesical pressure recording.

Results: Intradetrusor injection of BoNT-A for 1-2 weeks or 1 month significantly increased bladder capacity compared with saline injection ( $p<0.05$, two-way ANOVA). Following BoNT-A, SNS attenuated the frequency of bladder contractions, either eliminating bladder contractions or reducing the contraction frequency during electrical stimulation. Inhibition of the contraction frequency by SNS following BoNT-A treated rats was not different from that measured following saline injection.

Conclusions: BoNT-A increased the bladder capacity, but compensating for additional saline infusion to the enlarged urinary bladder in BoNT-A pretreated rats, the bladder contractions induced by bladder filling were attenuated by SNS. BoNT-A did not alter the ability of SNS to inhibit bladder contraction following intradetrusor injection of BoNT-A for 2 days, 1-2 weeks or 1 month. These results support further pre-clinical and clinical studies to evaluate potential interactions or combination therapy with neuromodulation and intradetrusor BoNT-A therapeutic approaches.

Keywords: Electrical stimulation, Micturition, Spinal nerve, Botulinum toxin

\section{Background}

Detrusor overactivity is a common medical problem resulting in increased voiding frequency, urinary urgency and urge incontinence. Anti-muscarinic drugs are generally used as first line therapies while mirabegron is a first-in-class $\beta 3$-adrenoceptor agonist licensed and has shown to be effective and have a good safety profile [1]. If medications cannot be tolerated or do not provide

\footnotetext{
* Correspondence: xin.su@medtronic.com

${ }^{1}$ Medtronic plc, Neuromodulation Research, 7000 Central Avenue, Minneapolis, MN 55432, USA

Full list of author information is available at the end of the article
}

sufficient symptomatic relief, the most common second line treatment options are 1) sacral neuromodulation (InterStim ${ }^{\circ}$ Therapy) via stimulation of the spinal nerve (S3), which is efficacious in 70-80\% population of patients with urge incontinence and increased frequency [2]; and 2) intradetrusor injection of botulinum toxin type A (BoNT-A) which improves detrusor overactivity and increases bladder capacity $[3,4]$.

Urodynamic studies have shown BoNT-A injection to increase maximum cystometric capacity and bladder compliance [5-10]. With the increasing use of BoNT-A, many patients may receive both of these second line 
therapies. Retrospective study has shown that patients who are dissatisfied with or fail BoNT-A treatment with a mean interval of 23 months between the BoNT-A and the sacral neuromodulation test stimulation, respond successfully to InterStim Therapy [11]. Systematic studies of potential interactions of sacral neuromodulation and BoNT-A have not been performed. One unknown question relates to the potential impact of prior BoNT-A treatment upon subsequent neuromodulation. Specifically, does prior treatment with BoNT-A alter patient success rates to neuromodulation therapy? No clinical or preclinical data is available to address this question from either efficacy or safety perspectives.

We have utilized the rat model of the bladder micturition reflex contraction (BRC) to screen for potential interactions between BoNT-A and neuromodulation [12-14]. Spinal nerve stimulation (SNS) results in an inhibition of the frequency of BRC. Stimulation parameters (e.g. stimulation frequency and current intensity) have been optimized systemically. Our previous work in the rat $[15,16]$ demonstrates a positive intensitydependent bladder inhibitory effect in response to electrical stimulation of the L6 spinal nerve. In the present study, we characterized the relationship between current intensity of SNS and bladder contraction frequency in rats pretreated with intradetrusor injection of either saline or BoNT-A.

\section{Methods}

\section{Animals and study design}

A total of 79 female Sprague-Dawley rats, 200-300 g were used, 44 in the BoNT-A treatment group (intradetrusor injection) and 35 in the saline treatment group. After 2 days to 1 month of the treatment, all rats were anesthetized with urethane and examined urodynamically to determine the bladder compliance or bladder micturition reflex following the treatment. The inhibitory effects of SNS on BRC were also studied. During the urodynamic study, rats were maintained at $37{ }^{\circ} \mathrm{C}$ with a heating pad (Viking Medical, Medford Lakes, NJ) and were euthanized by $\mathrm{CO}_{2}$ asphyxia upon completion of experimental procedures. The experimental protocols were approved by the Medtronic Institutional Animal Care and Use Committee as well as the Non-clinical Research Board (Minneapolis, MN).

\section{Intradetrusor injection}

Rats were anesthetized with $3 \%$ isoflurane. The urinary bladder was exposed via a small suprapubic incision under sterile conditions. Two units $(0.2 \mathrm{ml})$ of BoNT-A (BOTOX ${ }^{\circ}$, manufactured by Allergan, Besse Medical, West Chester, $\mathrm{OH}$ ) or saline were injected into the whole detrusor randomly and evenly $(10-16 \mu \mathrm{l}$ per site) through a 30 -gauge needle. The same volume of normal saline was injected into the detrusor at the same positions as a control group. The rectus fascia was closed with an absorbable suture, and the skin was closed with silk sutures. Animals then were housed for 2 days (9 in saline and 10 in BoNT-A), 1 week (11 in saline and 15 in BoNT-A) or 2 weeks (4 in saline and 6 in BoNT-A, grouped together with 1 week treatment), or 1 month (11 in saline and 13 in BoNT-A) before the urodynamic studies.

\section{Neuromodulation of L6 SNS}

Rats were anesthetized with urethane (two i.p. injections, $4 \mathrm{~min}$ apart, total $1.2 \mathrm{~g} / \mathrm{kg}, 200 \mathrm{mg} / \mathrm{ml}$ in saline, SigmaAldrich, St. Louis, MO). To deliver electrical stimulation, the skin around the dorsal sacral and thoracic area was shaved and a dorsal midline incision was made from approximately L3 to S2, the L6/S1 posterior processes were exposed. The $\mathrm{S} 1$ processes were removed and the L6 nerve trunks were localized caudal and medial to the sacroiliac junction. Electrodes were made from tefloncoated, 40-gauge, stainless steel wire with the coating removed from a $0.5 \mathrm{~cm}$ section (Cooner Wire Co., Chatsworth, CA) and was placed under each of the L6 spinal nerve bilaterally. After the wire electrode(s) were positioned, silicone adhesive (Kwik-Cast, World Precision Instruments, Inc, Fl, USA) was applied to cover the wire around the nerve, and the skin incision was sutured shut. The electrode(s) were connected to a Grass S88 stimulator (Grass Medical Instruments), through stimulus isolation unit(s) (SIU-BI, Grass Medical Instruments). A needle electrode under the skin of the tail served as the ground.

To record bladder contractions, a polyethylene cannula (PE50) was inserted into the bladder via the urethra, and secured with a suture tie. The urethral cannula was connected via a T- connector to a pressure transducer (MLT0380D, ADInstruments, Colorado Springs, CO, USA) and data acquisition system (ML880/P, ADInstruments). The intravesical pressure signal was amplified for recording (ML228, ADInstruments). The other end of the T-connector was attached to a syringe pump.

\section{Urodynamic studies}

To induce BRC, saline was infused into the bladder via the syringe pump at a rate of $50 \mu \mathrm{l}$ per minute to induce a micturition reflex (here defined as a bladder contraction of a magnitude $>10 \mathrm{mmHg}$ ). The infusion rate was then lowered to $10 \mu \mathrm{l}$ per minute and continued until 3-5 consecutive contractions were established. At this time, the saline infusion was terminated, and the BRC continued.

Electrical stimulation of the spinal nerve evoked hindtoe twitches and/or pelvic floor muscle contraction. In 
each rat, the motor threshold current $\left(\mathrm{T}_{\mathrm{mot}}\right)$ was defined as the lowest current required to evoke the first, barely observable, muscle contraction. Biphasic pulses (pulse width $0.1 \mathrm{~ms})$ of different intensities $\left(0.8 \times \mathrm{T}_{\text {mot }}, \mathrm{T}_{\text {mot }}\right.$ and $\left.2 \times \mathrm{T}_{\text {mot }}\right)$ were applied at a frequency of $10 \mathrm{~Hz}$ to stimulate the spinal nerve.

After a 15 min control period, nerve stimulation was applied; the BRC were recorded for additional $20 \mathrm{~min}$ post stimulation. Initially, single current intensity stimulation was applied for a total of $10 \mathrm{~min}$ (20 in saline and 25 in BoNT-A) in one rat. In some other experiments (15 in saline and 19 in BoNT-A), all intensities in the order $0.8 \times \mathrm{T}_{\text {mot }}, \mathrm{T}_{\text {mot }}$ and $2 \times \mathrm{T}_{\text {mot }}$ for a total of $15 \mathrm{~min}$ were tested in the same rat. Since there is no difference in sensitivity to nerve stimulation between two stimulation modalities [14-16], the data were grouped according the test intensity.

\section{Data analysis}

To evaluate the bladder compliance/capacity, a few cystometry parameters were assessed once the first micturition reflex was established including basal bladder pressure (BP, $\mathrm{mmHg}$ ), threshold pressure (TP, $\mathrm{mmHg}$, pressure before the micturition occurred), and bladder capacity ( $\mathrm{ml}$, volume infused to induce a micturition reflex, interval x $0.05 \mu \mathrm{l} \bullet \mathrm{min}-1)$. The bladder compliance was assessed mathematically using the capacity divided by the difference of BP with TP. Once the stable BRC is established, two parameters of BRC were evaluated: frequency/interval and amplitude between the maximum pressure reached during voiding and after voiding in $\mathrm{mmHg}$ in $5 \mathrm{~min}$ bins. When the BRC was completely suppressed by high intensity stimulation, the frequency of the bladder contraction was designated "0" and amplitude data for that interval was excluded. All data were compared to the mean response during the last $5 \mathrm{~min}$ prior to stimulation.

All data are expressed as mean \pm SEM. Results were analyzed with two-way ANOVA with Bonferroni posthoc test by Prism 5 (GraphPad Software, Inc., San Diego, CA). A value of $p<0.05$ was considered statistically significant.

\section{Results}

Following treatment of BoNT-A or saline, infusion of saline into the bladder at a constant rate induced an increase in bladder pressure. Until the TP for urination is reached, only a small increase in pressure was observed. Figure $1 \mathrm{a}$ and $\mathrm{b}$ shows the two traces of intravesical pressure from rats 1 month post intradetrusor injection of either saline or BoNT-A. More saline infusion was required to evoke the bladder micturition reflex in the rat pretreated with BoNT-A. Summarized data in Fig. 1c demonstrate that intradetrusor injection of BoNT-A for 1-2 weeks or 1 month significantly increased bladder capacity $(p=0.008$, two-way ANOVA). We found no differences on compliance, TP and BP between the control and BoNT-A groups (Fig. 1d, e and f). Continuing the saline infusion (see Methods) resulted in BRC. Once the BRC was established with large volume infusion in BoNT-A treated rats, the baseline values of contraction amplitude and contraction frequency from BoNT-A treated rats were not different from that from saline treated rats (Fig. $1 \mathrm{~g}$ and $\mathrm{h}$ ).

Without stimulation the BRC was similar following saline or BoNT-A pretreatment (Fig. 2a and c). When electrical stimulation of the spinal nerve was delivered it attenuated the frequency of bladder contractions, either eliminating bladder contractions completely or reducing the contraction frequency during electrical stimulation in both BoNT-A and saline treatments (Fig. $2 \mathrm{~b}$ and d). The contraction frequency returned to the prestimulation value after termination of electrical stimulus.

Figure 3 summarizes the mean responses of BRC responses during different intensities of SNS in saline or BoNT-A treated rats. Two-way ANOVA analysis demonstrates that BRC frequency was significantly decreased by SNS at all current intensities tested following either BoNT-A or saline injections $(p<0.05$, vs time control when stimulation was not applied).

Two days, 1-2 weeks and 1 month post BoNT-A injection, SNS at $\mathrm{T}_{\text {mot }}, 10 \mathrm{~Hz}$ decreased the frequency of contractions to $70.18 \pm 25 \%(\mathrm{n}=8), 52.14 \pm 12 \%(\mathrm{n}=16, p<$ $0.05)$, and $51.94 \pm 12 \%$ of controls $(\mathrm{n}=12, p<0.05$, vs. $98.77 \pm 5 \%$ control without stimulation), respectively. Using the same stimulation parameters, SNS decreased the frequency of contractions in saline pretreated rats, to $50.32 \pm 12 \%(\mathrm{n}=8, p<0.05), 55.93 \pm$ $15 \%(\mathrm{n}=11, p<0.05)$, and $45.45 \pm 10 \%$ of controls $(\mathrm{n}=9, \quad p<0.05$, vs. $114.16 \pm 8 \%$ control without stimulation), respectively. Inhibition of the contraction frequency in BoNT-A treated rats was not different from that measured in saline treated rats $(p>0.05)$.

SNS elicited relatively weak and inconsistent inhibition on the amplitude of the bladder contractions $(p<0.05$, vs time control when stimulation was not applied, twoway ANOVA). Figure 3b, $d$ and $f$ summarize effects of SNS on amplitude of BRC.

\section{Discussion}

We have previously reported that SNS inhibits the frequency of volume induced BRC, and that $10 \mathrm{~Hz}$ is the optimally efficacious stimulation frequency in this model system [15]. The inhibitory effects of SNS on bladder contractions have also been shown to be intensitydependent, and the magnitude of the inhibition increases with the applied current (stimulus intensity, 16). In the present study, we used $10 \mathrm{~Hz}$ stimulation frequency and 


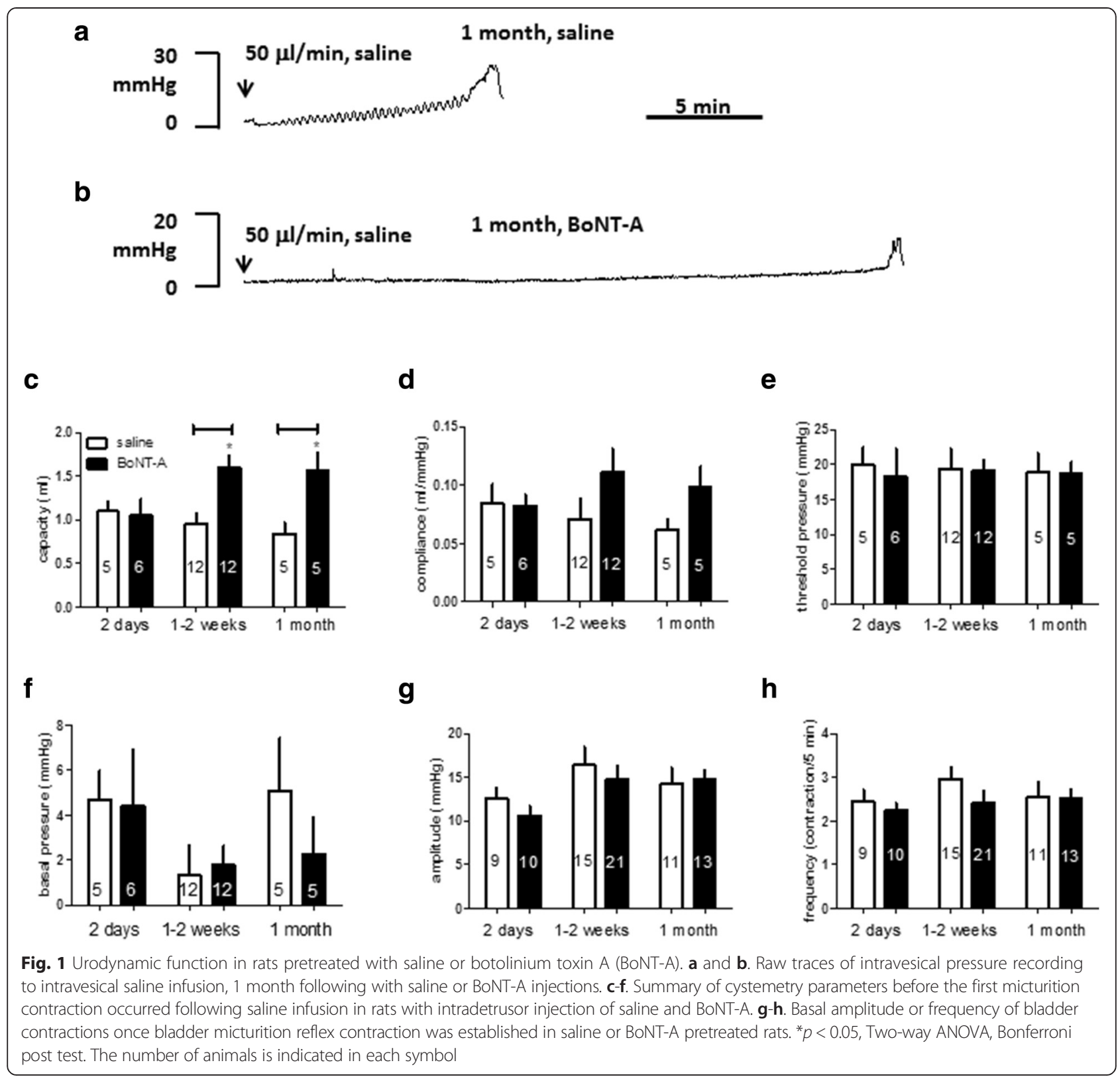

studied the intensity-response function of the bladder micturition reflex to SNS in BoNT-A or saline treated rats to establish the sensitivity of neuromodulation in bladder control. Our data do not show a difference in the magnitude of the inhibitory effect of neuromodulation between BoNT-A and saline treated rats.

Intradetrusor injection of the BoNT-A dose used in our study (2 units) was shown in a prior study to not cause urinary retention in the rat [8]. Consistent with these results, we did not observe any reduction in the amplitude of bladder contraction following BoNT-A treatment. The interval between BoNT-A injection and urodynamic evaluation ranged from 2 days to 1 month. BoNT-A significantly increased bladder capacity, 1-2 weeks and 1 month following injection. This is consistent with the time-course for the onset and duration of the effect of BoNT-A by others. Significant inhibitory effects on neurotransmitter release and urodynamic function were observed 5-30 days after injection [7, 17]. Clinically, patients experience relief of sudden urges to urinate, and a reduction in urine leakage starting several days to 2 weeks following BoNT-A injection. In patients the effect has been show to persist for 3 to 9 months [18].

In our tests the effectiveness of BoNT-A alone was demonstrated by its action on increasing bladder capacity. This is consistent with studies reported by others regarding effects of BoNT-A alone in the rat with 


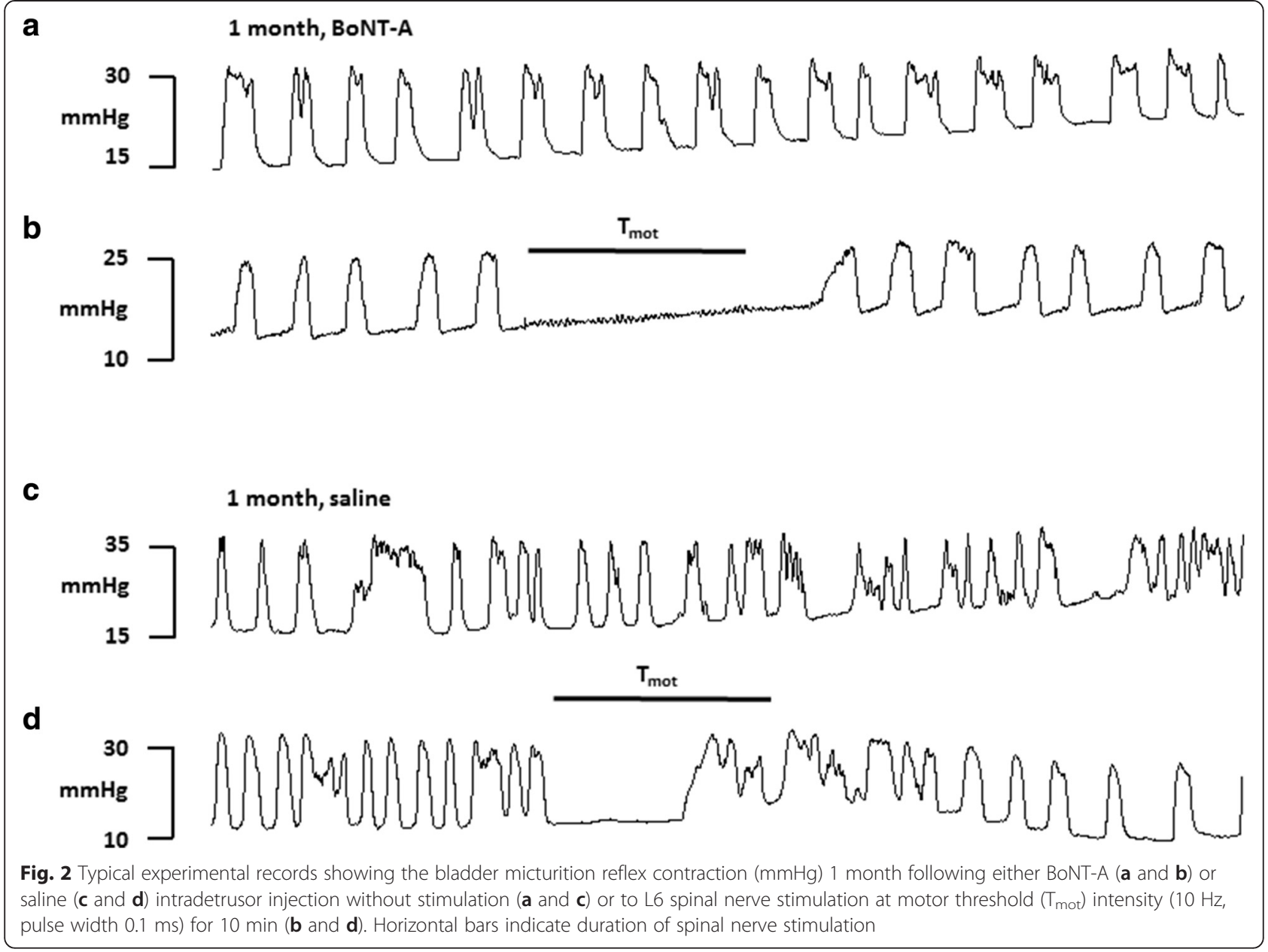

different manipulations $[6,8,9]$. In spinal cord injured rats, BoNT-A attenuated non-voiding contractions [9]. In addition, BoNT-A has been also reported to suppress acetic acid induced bladder hyperactivity and inflammatory reactions $[6,8]$. We did not examine the immunohistochemistry and histology changes on the detrusor following BoNT-A injection in the present study. Others have reported that the mechanism of BoNT-A on bladder control may be related to its inhibition on neurotransmitter release [17, 19-22]. This is reflected by an increase in bladder capacity. Clinically BoNT-A results in an increase in cystometric capacity. In studies reported increases of $60 \%$ ([23], spinal cord injured patients with detrusor overactivity), 35-80 \% ([24], pediatric neurogenic bladder), and 100-200 \% ([25], neurogenic detrusor overactivity patients) have been observed.

Intradetrusor injection of BoNT-A increases bladder capacity. Additional saline infusion was able to compensate the enlarged urinary bladder in BoNT-A pretreated rats and establish the BRC. Although the bladder capacity is increased, the frequency of bladder contraction in our model is not decreased, and the ability of neuromodulation to inhibit $\mathrm{BRC}$ is unaffected. In the present study, SNS produced an equal degree of bladder inhibition following either saline or BoNT-A treatment. The magnitude of inhibition was comparable to that reported previously in this model $[15,16]$. Therefore, in BoNT-A treated rats, SNS produced a bladder inhibition that occurred independent of the increased bladder capacity produced by BoNT-A. It is likely that BoNT-A acts via interference with the release of neurotransmitters from peripheral nerve terminals and the response of afferent activated dorsal horn neurons [26, 27] while neuromodulation produced an inhibition of the BRC via an action at a higher level CNS on the neuronal control of the bladder reflex (e.g. the pontine micturition center and cerebral cortex, [28]). If BoNT-A and SNS have different sites of action, it would be logical that these therapies may have independent effects on bladder inhibition.

The present results are not definitive. Detrusor overactivity is often a multi-etiological disease. Our data were obtained in normal and anesthetized rats. Only BRC, not cystometric capacity was measured. However, inhibition of the BRC is a consequence of an increased 


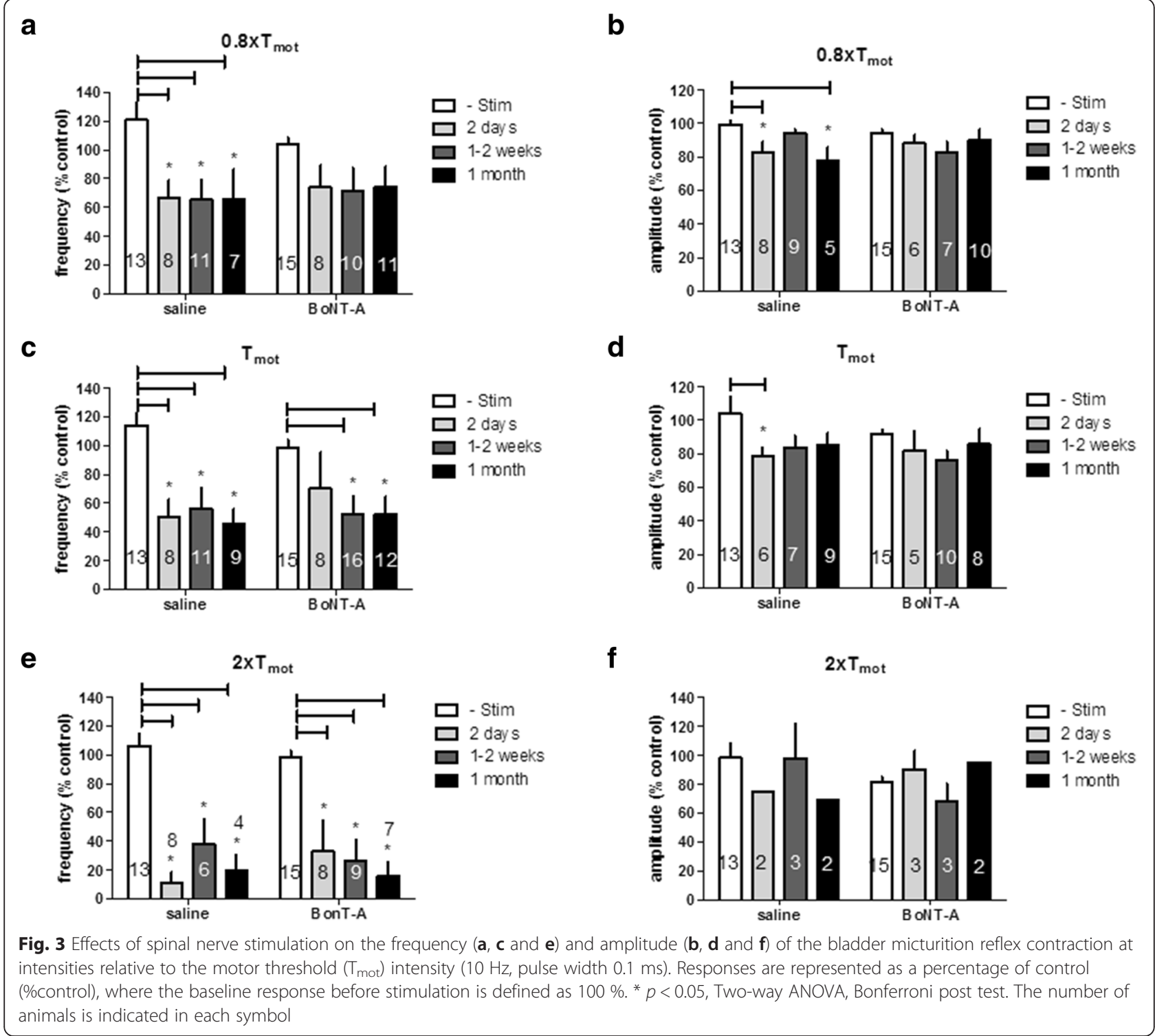

bladder capacity [29]. Since pretreatment with BoNTA does not appear to attenuate the bladder inhibitory effects of neuromodulation, it is reasonable that an equally increased bladder capacity would be observed in rats pretreated with intradetrusor injection of either BoNT-A or saline. In support, from a clinical observation patients who are dissatisfied with or fail BoNT-A treatment respond successfully to InterStim Therapy [11]. The neuromodulation was evaluated following a single injection of BoNT-A in this work. Our findings encourage further pre-clinical studies evaluating the combination of neuromodulation and repeated intradetrusor injections of BoNT-A on urodynamics in animals with normal and irritated bladder as well as controlled clinical evaluations of therapy interactions or combinations using sacral neuromodulation with BoNT-A in patients with detrusor overactivity.

\section{Conclusions}

Our study confirmed previous observations that intradetrusor injection of BoNT-A increases bladder capacity in the rat. This increased capacity in BoNT-A treated rats does not influence the frequency of the BRC. Regardless of the interval between injection and stimulation, treatment with BoNT-A does not appear to influence the ability of SNS to inhibit BRC. In future studies it will be important to test whether neuromodulation is also efficacious in BoNT-A treated patients, and whether the two treatments could be combined in the treatment of patients with refractory detrusor overactivity. 


\section{Abbreviation}

BoNT-A: Botulinum toxin A; BRC: Bladder rhythmic contraction; SNS: Spinal nerve stimulation; $T_{\text {mot: }}$ : Motor threshold; BP: Basal bladder pressure; TP: Threshold pressure.

\section{Competing interests}

All authors are employees of the Medtronic, plc. The research was funded by Medtronic, plc.

\section{Authors' contributions}

XS designed the study, performed data analysis and drafted the manuscript AN performed the experiment, DEN helped to draft the manuscript. All authors read and approved the final manuscript.

\section{Acknowledgements}

The authors are grateful to Dr. Greg Molnar for helpful comments and Dr. Matt Kelly for study coordination. The manuscript was edited by J. Paul Hieble Scientific Writing.

\section{Author details}

'Medtronic plc, Neuromodulation Research, 7000 Central Avenue, Minneapolis, MN 55432, USA. ${ }^{2}$ Physiological Research Laboratories, 7000 Central Avenue, Minneapolis, MN 55432, USA.

Received: 5 December 2014 Accepted: 3 June 2015

\section{Published online: 09 June 2015}

\section{References}

1. Maman K, Aballea S, Nazir J, Desroziers K, Neine ME, Siddiqui E, et al. Comparative efficacy and safety of medical treatments for the management of overactive bladder: a systematic literature review and mixed treatment comparison. Eur Urol. 2014;65:755-65.

2. Hussain Z, Harrison SWW. Neuromodulation for lower urinary tract dysfunction - an update. Sci World J. 2007;7:1036-45.

3. Apostolidis A, Dasqupta P, Denys P, Elneil S, Fowler CJ, Giannantoni A, et at. Recommendations on the use of botulinum toxin in the treatment of lower urinary tract disorders and pelvic floor dysfunctions: a European consensus report. Eur Urol. 2009;55:100-20.

4. Smith $\mathrm{CP}$. Botulinum toxin in the treatment of $\mathrm{OAB}, \mathrm{BPH}$, and IC. Toxicon. 2009:54:639-46.

5. Smith CP, Boone TB, de Groat WC, Chancellor MB, Somogyi GT. Effect of stimulation intensity and botulinum toxin isoform on rat bladder strip contractions. Brain Res Bull. 2003;61:165-71.

6. Atiemo H, Wynes J, Chuo J, Nipkow L, Sklar GN, Chai TC. Effect of botulinum toxin on detrusor overactivity induced by intravesical adenosine triphosphate and capsaicin in a rat model. Urology. 2005:65:622-6.

7. Krhut J, Zvara P. Intravesical instillation of botulinum toxin A: an in vivo murine study and pilot clinical trial. Int Urol Nephrol. 2011:43:337-43.

8. Cayan S, Coşkun B, Bozlu M, Acar D, Akbay E, Ulusoy E. Botulinum toxin type A may improve bladder function in a rat chemical cystitis model. Urol Res. 2003:30:399-404.

9. Behr-Roussel D, Oger S, Pignol B, Pham E, Le Maux A, Chabrier PE, et al. Minimal effective dose of dysport and botox in a rat model of neurogenic detrusor overactivity. Eur Urol. 2012;61:1054-61.

10. Khera M, Somogyi GT, Salas NA, Kiss S, Boone TB, Smith CP. In vivo effects of botulinum toxin $\mathrm{A}$ on visceral sensory function in chronic spinal cord-injured rats. Urol. 2005:66:208-12.

11. Smits MA, Oerlemans D, Marcelissen TA, Van Kerrebroeck PE, De Wachter SG. Sacral neuromodulation in patients with idiopathic overactive bladder after initial botulinum toxin therapy. J Urol. 2013;190:2148-52.

12. Su X, Nickles A, Nelson DE. Comparison of neural targets for neuromodulation of bladder micturition reflex in the rat. Am J Physiol Renal Physiol. 2012;303:F1196-206.

13. Su X, Nickles A, Nelson DE. Quantification of effectiveness of bilateral and unilateral neuromodulation in the rat bladder rhythmic contraction model. BMC Urol. 2013:13:34.

14. Su X, Nickles A, Nelson DE. Differentiation and interaction of tibial versus spinal nerve stimulation for micturition control in the rat. Neurourol Urodyn. 2015;34:92-7.

15. Su X, Nickles A, Nelson DE. Neuromodulation in a rat model of the bladder micturition reflex. Am J Physiol Renal Physiol. 2012;302:F477-86.
16. Su X, Nickles A, Nelson DE. The role of the endogenous opioid system in modulation of urinary bladder activity by spinal nerve stimulation. Am J Physiol Renal Physiol. 2013;305:F53-60

17. Smith CP, Franks ME, McNeil BK, Ghosh R, de Groat WC, Chancellor MB, et al. Effect of botulinum toxin $A$ on the autonomic nervous system of the rat lower urinary tract. J Urol. 2003;169:1896-900.

18. Granese R, Adile G, Gugliotta G, Cucinella G, Saitta S, Adile B. Botox $\left({ }^{(}\right)$for idiopathic overactive bladder: efficacy, duration and safety. Effectiveness of subsequent injection. Arch Gynecol Obstet. 2012;286:923-9.

19. Smith $C P$, Gangitano DA, Munoz A, Salas NA, Boone TB, Aoki KR, et al. Botulinum toxin type A normalizes alterations in urothelial ATP and NO release induced by chronic spinal cord injury. Neurochem Int. 2008;52:1068-75.

20. Lawrence GW, Aoki KR, Dolly JO. Excitatory cholinergic and purinergic signaling in bladder are equally susceptible to botulinum neurotoxin a consistent with co-release of transmitters from efferent fibers. J Pharmacol Exp Ther. 2010;334:1080-6.

21. Lucioni A, Bales GT, Lotan TL, McGehee DS, Cook SP, Rapp DE. Botulinum toxin type A inhibits sensory neuropeptide release in rat bladder models of acute injury and chronic inflammation. BJU Int. 2008;101:366-70.

22. Rapp DE, Turk KW, Bales GT, Cook SP. Botulinum toxin type a inhibits calcitonin gene-related peptide release from isolated rat bladder. J Urol. 2006;175:1138-42.

23. Schurch B, Stöhrer M, Kramer G, Schmid DM, Gaul G, Hauri D. Botulinum-A toxin for treating detrusor hyperreflexia in spinal cord injured patients: a new alternative to anticholinergic drugs? Preliminary results. J Urol. 2000;164:692-7.

24. Sager C, Burek C, Durán V, Corbetta JP, Weller S, Juan B, et al, Pharmacotherapy in pediatric neurogenic bladder intravesical botulinum toxin type A. ISRN Urol. 2012;2012:763159.

25. Patel AK, Patterson JM, Chapple CR. Botulinum toxin injections for neurogenic and idiopathic detrusor overactivity: A critical analysis of results. Eur Urol. 2006:50:684-709

26. Elkelini MS, Bagli DJ, Fehlings M, Hassouna M. Effects of intravesical onabotulinumtoxinA on bladder dysfunction and autonomic dysreflexia after spinal cord injury: role of nerve growth factor. BJU Int. 2012;109:402-7.

27. Munoz A, Somogyi GT, Boone TB, Smith CP. Central inhibitory effect of intravesically applied botulinum toxin A in chronic spinal cord injury. Neurourol Urodyn. 2011;30:1376-81.

28. Noblett KL, Cadish LA. Sacral nerve stimulation for the treatment of refractory voiding and bowel dysfunction. Am J Obstet Gynecol. 2014;210:99-106.

29. Su X, Nickles A, Nelson DE. Neuromodulation attenuates bladder hyperactivity in a rat cystitis model. BMC Urol. 2013;13:70.

\section{Submit your next manuscript to BioMed Central and take full advantage of:}

- Convenient online submission

- Thorough peer review

- No space constraints or color figure charges

- Immediate publication on acceptance

- Inclusion in PubMed, CAS, Scopus and Google Scholar

- Research which is freely available for redistribution 